\title{
Evaluation of the in vitro antibacterial activity of some essential oils and their blends against Staphylococcus spp. isolated from episodes of sheep mastitis
}

\author{
Filippo Fratini $^{1,2} \mathbb{D} \cdot$ Margherita Giusti $^{3} \cdot$ Simone Mancini $^{1} \mathbb{D} \cdot$ Francesca Pisseri $^{3} \cdot$ Basma Najar $^{4}(\mathbb{D}) \cdot$ Luisa $^{\text {Pistelli }}{ }^{2,4} \mathbb{( D}$
}

Received: 19 February 2021 / Accepted: 16 April 2021 / Published online: 11 May 2021

(C) The Author(s) 2021

\begin{abstract}
Staphylococcus aureus and coagulase-negative staphylococci are among the major causes of mastitis in sheep. The main goal of this research was to determine the in vitro antibacterial activity of several essential oils (EOs, $n$ 30), then five of them were chosen and tested alone and in blends against staphylococci isolates. Five bacteria were isolated from episodes of ovine mastitis (two S. aureus and three S. xylosus). Biochemical and molecular methods were employed to identify the isolates and disk diffusion method was performed to determine their antimicrobial-resistance profile. The relative percentage of the main constituents in the tested essential oils and their blends was detected by GC-EIMS analysis. Antibacterial and bactericidal effectiveness of essential oils and blends were evaluated through minimal inhibitory concentration (MIC) and minimal bactericidal concentration (MBC). All of them showed sensitivity to the used antimicrobials. The EOs with the highest antibacterial activity were those belonging to the Lamiaceae family characterized by high concentrations of thymol, carvacrol and its precursor $p$-cymene, together with cinnamon EO, rich in cinnamaldehyde. In terms of both MIC and MBC values, the blend composed by Thymus capitatus EO 40\%, Cinnamomum zeylanicum EO 20\%, Thymus serpyllum EO $20 \%$ and Satureja montana EO 20\% was found to be the most effective against all the isolates. Some essential oils appear to represent, at least in vitro, a valid tool against ovine mastitis pathogens. Some blends showed a remarkable effectiveness than the single oils, highlighting a synergistic effect in relation to the phytocomplex.
\end{abstract}

Keywords Cinnamon bark · Everlasting flowers · Winter savory $\cdot$ Thyme $\cdot$ Synergy

\section{Introduction}

Filippo Fratini and Margherita Giusti equally contributed to this work.

Filippo Fratini

filippo.fratini@unipi.it

Margherita Giusti

anguilla3@virgilio.it

Simone Mancini

simone.mancini@unipi.it

Francesca Pisseri

info@francescapisseri.it

Basma Najar

basmanajar@hotmail.fr

Luisa Pistelli

luisa.pistelli@unipi.it
Mastitis is one of the most important health problem in dairy sheep worldwide. Clinical mastitis incidence during lactation is usually less than 5\%, but, in some cases, it can reach or overcome $30 \%$ (Contreras et al. 2007). Staphylococcus

1 Department of Veterinary Sciences, University of Pisa, Viale Delle Piagge 2, Pisa, Italy

2 Interdepartmental Research Center "Nutraceuticals and Food for Health", University of Pisa, Via del Borghetto 80, Pisa, Italy

3 Veterinary practitioner, Pisa, Italy

4 Department of Pharmacy, University of Pisa, Via Bonanno 6, Pisa, Italy 
aureus and coagulase-negative staphylococci (CoNS) are considered, respectively, as the main pathogens involved in clinical and subclinical mastitis in sheep (Bergonier et al. 2003; Mork et al. 2005; Contreras et al. 2007). The increasing concern about antimicrobial resistance among strains of these bacteria represents a great challenge for research, to find alternative, safe and innovative strategies, as the use of phytochemicals and essential oils (EOs) (Sharma et al. 2018).

The antimicrobial effects of EOs originated from medicinal and aromatic plants are relevant, since they may inhibit the growth of Staphylococcus strains or kill bacterial cells (Fratini et al. 2014; Tariq et al. 2019) both if used alone or in combination (Fratini et al. 2017; van Vuuren et al. 2019). The phytocomplex contained in a single EO, which consists of compounds of several different functional-group classes, can be used alone or in association with other phytocomplexes providing a multiplicity of pharmacological actions, related to the presence of active compounds that may perform various functions and others that modulate the effect (Pisseri et al. 2008; Marassi and Rossi 2015). Thymus capitatus, Thymus serpyllum and Satureja montana belong to the Lamiaceae family, which includes plants with a wide range of biological and pharmacological activities (Marin et al. 2018). Essential oils and extracts from the genus Thymus exhibited different biological properties such as antioxidant, antibacterial, antifungal, antiviral, antiparasitic, cytotoxic, carminative and spasmolytic (Paaver et al. 2008; Nabavi et al. 2015; Maissa and Walid 2015). EOs from various Satureja species as well have demonstrated antibacterial, antiviral, antiparasitic, antioxidant, anti-inflammatory, antinociceptive, hepatoprotective, antidiabetic and anticholesterolemic activities (Jafari et al. 2016; Caprioli et al. 2019). The antibacterial activity has been attributed to the presence of oxygenated monoterpenes, especially thymol and carvacrol in synergy with its precursor $p$-cymene (Jarić et al. 2015; Tepe and Cilkiz 2016). These compounds showed to be effective against $S$. aureus and CoNS (Hyldgaard et al. 2012; dos Santos Rodrigues et al. 2017; Gaio et al. 2017).

The genus Cinnamomum consists of over 250 aromatic trees and shrubs members of the Lauraceae family, distributed in warm temperate and tropical regions. The main components of Cinnamomum zeylanicum EO are monoterpenes, sesquiterpenes and their related derivatives, mostly cinnamaldehyde obtained from bark and eugenol from leaves (Barceloux 2009). This plant extract showed antimicrobial properties against Staphylococcus spp., also in association with antibiotics (Dal Pozzo et al. 2012; Mahadlek et al. 2012; Saleem et al. 2015).

Helychrysum italicum (Asteraceae), a Mediterranean aromatic shrub, EO exhibits a marked anti-inflammatory and antimicrobial properties against Gram-positive bacteria (Demir et al. 2009; Antunes Viegas et al. 2014; Djihane and
Mihoub 2016), along with an elevated capacity to increase the antibiotics effectiveness against multidrug-resistant Gram-negative bacteria (Lorenzi et al. 2009). Water or steam distillation of the aerial parts produced a mixture of phytochemicals mainly rich in monoterpenes and sesquiterpenes, followed by $\beta$-diketones (Maksimovic et al. 2017).

The aim of this research was to screen by in vitro tests the antibacterial activity of some essential oils. The EOs were selected on the basis of the available scientific literature, but also taking into account their synergistic activity encountered during personal field experience. Then, the most effective five, were tested alone and in blends against staphylococci isolated from episodes of sheep mastitis.

\section{Materials and methods}

\subsection{Farm description and sampling}

Casorelle is a multifunctional agrozootechnical farm $\left(43^{\circ} 49^{\prime} 43,5^{\prime \prime} \mathrm{N}-10^{\circ} 54^{\prime} 26,2^{\prime \prime} \mathrm{E}\right)$ where Assaf sheep are reared in which the animals fed mainly forage through olive agroforestry system. Health management is focused on prevention and use of natural medicines such as phytotherapy and homeopathy. Forty-five individual milk samples, one for each udder half were collected from symptomatic and asymptomatic subjects during the period from April 2016 to June 2016 Table 1.

\subsection{Phenotypical and genotypical identification and antimicrobial-resistance profile of isolates}

All isolates presumptively imputable to mastitis etiological agents, were submitted to phenotypical and genotypical characterization according to Windria et al. (2016). Subsequently, the antibiotic resistance profile of each isolate was determined by disk diffusion method (Cockerill III et al. 2012), performing three replicates. Nineteen antimicrobials were tested (Table 2): amoxicillin-clavulanic acid (30 $\mu \mathrm{g}, \mathrm{AMC})$, amikacin $(30 \mu \mathrm{g}, \mathrm{AMK})$, ampicillin (10 $\mu \mathrm{g}, \mathrm{AMP})$, amoxicillin $(10 \mu \mathrm{g}, \mathrm{AMX})$, ceftazidime (30 $\mu \mathrm{g}$, CAZ), cephalothin (30 $\mu \mathrm{g}, \mathrm{CEF})$, ciprofloxacin $(5 \mu \mathrm{g}, \mathrm{CIP})$, cefotaxime (30 $\mu \mathrm{g}, \mathrm{CTX})$, doxycycline (30 $\mu \mathrm{g}$, DOX), enrofloxacin $(5 \mu \mathrm{g}, \mathrm{ENR})$, gentamicin $(10 \mu \mathrm{g}$, GEN $)$, cephalexin (30 $\mu \mathrm{g}, \mathrm{LEX})$, neomycin $(30 \mu \mathrm{g}, \mathrm{NEO})$, piperacillin (100 $\mu \mathrm{g}$, PIP), rifampin (30 $\mu \mathrm{g}$, RIF), streptomycin (10 $\mu \mathrm{g}, \mathrm{STR})$, trimethoprim-sulfamethoxazole (1.25-23.75 $\mu \mathrm{g}, \mathrm{SXT})$, tetracycline $(30 \mu \mathrm{g}, \mathrm{TET})$ and tobramycin $(10 \mu \mathrm{g}$, TOB). Antimicrobial disks were purchased from Oxoid Thermo Scientific (Milan, Italy).

The interpretation of the results was performed following EUCAST (2017) and CLSI (2013) guidelines. 
Table 1 Characteristics of the essential oils used in screening tests

\begin{tabular}{|c|c|c|c|c|c|}
\hline Order & Family & Specie & Parts of plant & Extraction method & Origin \\
\hline \multirow[t]{4}{*}{ Apiales } & \multirow[t]{4}{*}{ Apiaceae } & Anethum graveolens $\mathrm{L}$ & Whole plant during flowering & Steam distillation & Italy \\
\hline & & Coriandrum sativum $\mathrm{L}$ & Seeds & Steam distillation & Ukraine \\
\hline & & Cuminum cyminum $\mathrm{L}$ & Seeds & Steam distillation & Egypt \\
\hline & & Pimpinella anisum $\mathrm{L}$ & Seeds & Steam distillation & France \\
\hline \multirow[t]{2}{*}{ Astrales } & \multirow[t]{2}{*}{ Asteraceae } & Chamaemelum nobile All & Whole plant during flowering & Steam distillation & Italy \\
\hline & & Helichrysum italicum (Roth) G. Don & Whole plant during flowering & Steam distillation & Italy \\
\hline Illiciales & Illiciaceae & Illicium verum Hook.f & Fruits/seeds & Steam distillation & Vietnam \\
\hline \multirow[t]{10}{*}{ Lamiales } & \multirow[t]{10}{*}{ Lamiaceae } & Lavandula officinalis Chaix & Flowers & Steam distillation & Italy \\
\hline & & Ocimum basilicum $\mathrm{L}$ & Whole plant during flowering & Steam distillation & Egypt \\
\hline & & Origanum majorana $\mathrm{L}$ & Whole plant during flowering & Steam distillation & Egypt \\
\hline & & Origanum vulgare $\mathrm{L}$ & Whole plant during flowering & Steam distillation & Italy \\
\hline & & Rosmarinus officinalis $\mathrm{L}$ & Whole plant during flowering & Steam distillation & Italy \\
\hline & & Rosmarinus officinalis L. ct. CINEOLE & Whole plant during flowering & Steam distillation & Tunisia \\
\hline & & Satureja montana $\mathrm{L}$ & Whole plant during flowering & Steam distillation & Egypt \\
\hline & & Thymus capitatus (L.) Hoffmanns. \& Link & Whole plant during flowering & Steam distillation & Spain \\
\hline & & Thymus serpyllum $\mathrm{L}$ & Whole plant during flowering & Steam distillation & Turkey \\
\hline & & Thymus vulgaris L. ct. THYMOL & Whole plant during flowering & Steam distillation & Italy \\
\hline \multirow[t]{3}{*}{ Laurales } & \multirow[t]{3}{*}{ Lauraceae } & Cinnamomum zeylanicum Blume (60\%) & Bark & $\begin{array}{l}\text { Steam distillation } \\
\text { (40\% ethanol) }\end{array}$ & Germany \\
\hline & & Laurus nobilis $\mathrm{L}$ & Leaves & Steam distillation & Bosnia \\
\hline & & Litsea cubeba (Lour.) Pers & Fruits/leaves & STEAM distillation & China \\
\hline Myrtales & Myrtaceae & $\begin{array}{l}\text { Leptospermum scoparium J.R.Forst. \& } \\
\text { G.Forst }\end{array}$ & Flowers & Steam distillation & New Zealand \\
\hline \multirow[t]{3}{*}{ Pinales } & Cupressaceae & Juniperus communis $\mathrm{L}$ & Berries & Steam distillation & Albania \\
\hline & \multirow[t]{2}{*}{ Pinaceae } & Picea abies (L.) H. Karst & Tops & Steam distillation & France \\
\hline & & Pinus cembra $\mathrm{L}$ & Tops & Steam distillation & Austria \\
\hline Rosales & Rosaceae & Rosa $\times$ damascena Mill & Flowers & Solvent & Turkey \\
\hline \multirow[t]{4}{*}{ Sapindales } & \multirow[t]{4}{*}{ Rutaceae } & Cistus ladanifer $\mathrm{L}$ & Tops & Steam distillation & Spain \\
\hline & & Citrus $\times$ bergamia Risso \& Poit & Peel & Cold pressing & Italy \\
\hline & & Citrus limon (L.) Osbeck & Peel & Cold pressing & Argentina \\
\hline & & Citrus medica $\mathrm{L}$ & Fruits & Cold pressing & Italy \\
\hline Zingiberales & Zingiberaceae & Elettaria cardamomum (L.) Maton & Seeds & Steam distillation & Sri Lanka \\
\hline
\end{tabular}

\subsection{Essential oils and blends}

Thirty EOs were employed in the screening test (Table 1) provided by FLORA s.r.l. ® (Lorenzana, Pisa, Italy). EOs were selected on the basis of their antibacterial potential and their anti-inflammatory activities (i.e. everlasting flowers and rosemary). The inflammatory process in mammary gland enhances the development and progression of the bacterial infection; therefore, it is significantly advantageous to use a mixture that has both characteristics, antibacterial and anti-inflammatory, in the preparation of a therapeutic aid to be used in the clinical practice of ovine mastitis (Pisseri et al. 2008).

Basing on the EO antibacterial activities and the antiinflammatory properties, five blends (A, B, C, D and E) were constituted with Cinnamomum zeylanicum $(C z)$,
Helichrysum italicum (Hi), Satureja montana (Sm), Thymus capitatus (Tc) and Thymus serpyllum (Ts) EOs as following:

Blend A: Sm 30\%; Tc 30\%; Hi 25\%; Cz 15\%,

Blend B: Sm 30\%; Ts 25\%; Tc 25\%; $C z$ 20\%,

Blend C: Sm 34\%; Cz 34\%; Tc 22\%; Ts 10\%,

Blend D: Tc 50\%; Sm 30\%; Hi 20\%,

Blend E: Tc 40\%; $C z$ 20\%; Sm 20\%; Ts 20\%.

\subsection{Chemical characterization of essential oils and blends}

The analysis of the five EOs chosen and their blends was performed by GC-EIMS (gas chromatography-electron impact mass spectrometry) in Table 3 according to the method previously described by Pistelli et al. (2017). 
Table 2 Antimicrobial susceptibility test

\begin{tabular}{|c|c|c|c|c|c|c|c|}
\hline Class & Drug & Abbreviation & $S a 1$ & $\mathrm{Sa} 2$ & $S x 1$ & $S \times 2$ & $S \times 3$ \\
\hline \multirow[t]{4}{*}{ Penicillins } & Amoxicillin & AML & $\mathrm{S}$ & $\mathrm{S}$ & $\mathrm{R}$ & $\mathrm{R}$ & $\mathrm{R}$ \\
\hline & Amoxicillin-clavulanic acid & $\mathrm{AMC}$ & $\mathrm{S}$ & $\mathrm{S}$ & $\mathrm{S}$ & $\mathrm{S}$ & $\mathrm{R}$ \\
\hline & Ampicillin & AMP & $\mathrm{R}$ & $\mathrm{R}$ & $\mathrm{R}$ & $\mathrm{R}$ & $\mathrm{R}$ \\
\hline & Piperacillin & PRL & $\mathrm{S}$ & $\mathrm{S}$ & $\mathrm{S}$ & $\mathrm{S}$ & I \\
\hline \multirow[t]{4}{*}{ Cephalosporins } & Cephalexin & CL & I & $\mathrm{S}$ & $\mathrm{R}$ & I & $S$ \\
\hline & Cephalothin & $\mathrm{KF}$ & $S$ & $\mathrm{~S}$ & $S$ & $\mathrm{~S}$ & $S$ \\
\hline & Cephotaxime & CTX & I & I & I & $\mathrm{R}$ & I \\
\hline & Ceftazidime & CAZ & $\mathrm{S}$ & $\mathrm{S}$ & $\mathrm{R}$ & $\mathrm{R}$ & $\mathrm{R}$ \\
\hline \multirow[t]{2}{*}{ Fluoroquinolones } & Ciprofloxacin & CIP & I & $\mathrm{S}$ & $S$ & $\mathrm{~S}$ & $\mathrm{~S}$ \\
\hline & Enrofloxacina & ENR & I & $\mathrm{S}$ & $\mathrm{S}$ & I & $\mathrm{S}$ \\
\hline \multirow[t]{5}{*}{ Aminoglycosides } & Amikacin & $\mathrm{AK}$ & $\mathrm{S}$ & $\mathrm{S}$ & I & $\mathrm{R}$ & $\mathrm{S}$ \\
\hline & Neomycin & $\mathrm{N}$ & I & $\mathrm{S}$ & $\mathrm{R}$ & $\mathrm{S}$ & $\mathrm{S}$ \\
\hline & Streptomycin & $S$ & I & I & $\mathrm{R}$ & I & $\mathrm{S}$ \\
\hline & Gentamycin & $\mathrm{CN}$ & $\mathrm{S}$ & $\mathrm{S}$ & $S$ & $\mathrm{~S}$ & $\mathrm{~S}$ \\
\hline & Tobramycin & TOB & $S$ & $\mathrm{~S}$ & $S$ & I & $S$ \\
\hline \multirow[t]{2}{*}{ Tetracyclines } & Doxycycline & DO & $\mathrm{S}$ & $\mathrm{R}$ & I & I & $\mathrm{S}$ \\
\hline & Tetracycline & $\mathrm{TE}$ & $\mathrm{S}$ & $\mathrm{R}$ & $\mathrm{R}$ & $\mathrm{R}$ & $\mathrm{S}$ \\
\hline $\begin{array}{l}\text { Diaminopyrimi- } \\
\text { dines + Sulfona- } \\
\text { mides }\end{array}$ & Trimethoprim-sulfamethoxazole & SXT & $S$ & $\mathrm{~S}$ & $S$ & $\mathrm{~S}$ & $S$ \\
\hline Rifamycins & Rifampicin & RD & $\mathrm{S}$ & $\mathrm{S}$ & $\mathrm{S}$ & I & $\mathrm{S}$ \\
\hline
\end{tabular}

Staphylococcus aureus isolates: $S a 1$ and Sa2. Staphylococcus xylosus isolates: Sx1, Sx2 and Sx3

Antimicrobial susceptibility following EUCAST (2017) and CLSI (2013) guidelines determine as sensible (S), intermediate (I) and resistant (R)

\subsection{MIC and MBC determinations}

For each EO alone and, subsequently, for each mixture, MIC and MBC determinations were performed by the twofold serial microdilution method (Fratini et al. 2019) and expressed as w/v. Both assays were carried out in triplicate (Table 4).

\section{Results and discussion}

Only five individual milk samples out of forty-five (11.11\%) were positive for imputable mastitis etiological agents. Isolates were phenotypically and genotypically identified as Staphylococcus aureus (named Sa1 and Sa2) and Staphylococcus xylosus (named $S \times 3, S x 4$ and $S x 5$ ). Consistently with the existing literature on the frequency of the aetiological agents responsible for mastitis in sheep, our study led to the isolation of staphylococci specifically related to the species S. aureus and S. xylosus (Bergonier et al. 2003; Mork et al. 2005; Contreras et al. 2007). S. xylosus is one of the most frequently identified microorganisms in course of ovine mastitis (Vanderhaeghen et al. 2015; Cannas et al. 2019).

Antimicrobial-resistance profile of isolates is reported in Table 2. S. aureus isolates showed an overall antimicrobial susceptibility in contrast to Wendlandt et al. (2013).
Nevertheless, some studies proved that many S. aureus strains responsible for mastitis in livestock are characterized by high susceptibility to antibiotics, especially in those farms where limited use of these substances is made (Antonios et al. 2015; Lisowska-Łysiak et al. 2018). The three isolates belonging to $S$. xylosus specie showed heterogeneous results in terms of susceptibility to antimicrobials. This extreme variability was predictable in wild-type microorganisms of environmental origin are generally more often subjected to high selective pressure by various molecules and are characterized by a greater probability of horizontal transmission of genetic material (Pyorala and Taponen 2009; Taponen et al. 2015).

The main aim of this work was to evaluate potential alternatives to antimicrobial treatments of ovine mastitis, identifying which essential oils or their mixtures could represent an effective therapeutic tool. Seven out of thirty EOs analysed in the screening step showed a strong in vitro antibacterial activity (data not shown). In brief, Cinnamomum zeylanicum (mode values between 1:512 v/v and 1:1024 v/v), Leptospermum scoparium (mode values between 1:256 v/v and 1:1024 v/v), Thymus vulgaris thymol CT (mode values between 1:128 v/v and 1:512 v/v), Satureja montana (mode values between 1:128 v/v and 1:256 v/v), Origanum vulgare, Thymus serpyllum and Thymus capitatus (mode values between 1:256 v/v and 1:512 v/v) were the most effective. 
Table 3 The GC-EIMS analysis results of individual essential oils (EOs) and mixtures (relative abundance)

\begin{tabular}{|c|c|c|c|c|c|c|c|c|c|c|c|c|}
\hline \multirow[t]{2}{*}{ Compound } & \multirow[t]{2}{*}{ Class } & \multirow[t]{2}{*}{ LRI } & \multicolumn{5}{|l|}{ Eos } & \multicolumn{5}{|c|}{ Mixture of EOs } \\
\hline & & & $C z$ & $H i$ & $S m$ & $T s$ & $T c$ & A & B & $\mathrm{C}$ & $\mathrm{D}$ & $\mathrm{E}$ \\
\hline$\alpha$-Thujene & $\mathrm{mh}$ & 930 & 0.2 & - & 0.5 & 0.4 & 0.3 & 0.3 & 0.4 & 0.4 & 0.3 & 0.3 \\
\hline$\alpha$-Pinene & $\mathrm{mh}$ & 939 & 0.8 & - & 1.2 & 0.6 & 0.6 & 5.3 & 0.9 & 0.9 & 3.2 & 0.7 \\
\hline Camphene & $\mathrm{mh}$ & 954 & 0.3 & 0.6 & 0.7 & 0.2 & 0.1 & 0.4 & 0.4 & 0.4 & 0.2 & 0.3 \\
\hline Benzaldehyde & $\mathrm{Nt}$ & 960 & 0.2 & - & - & - & - & - & - & - & - & - \\
\hline Sabinene & $\mathrm{mh}$ & 975 & 0.4 & 0.5 & 0.6 & 0.2 & 0.1 & 0.4 & 0.4 & 0.4 & 0.4 & 0.3 \\
\hline 1-Octen-3-ol & $\mathrm{Nt}$ & 979 & - & - & 0.6 & 0.2 & 0.2 & 0.2 & 0.4 & 0.4 & 0.3 & 0.3 \\
\hline Myrcene & $\mathrm{mh}$ & 991 & 0.1 & - & 1.3 & 1.4 & 1.5 & 1.1 & 1.4 & 1.2 & 1.2 & 1.2 \\
\hline$\alpha$-Phellandrene & $\mathrm{mh}$ & 1003 & 1.0 & - & 0.3 & 0.1 & 0.2 & 0.2 & 0.3 & 0.4 & 0.2 & 0.3 \\
\hline$\delta$-3-Carene & $\mathrm{mh}$ & 1007 & 0.1 & - & - & - & - & - & 0.1 & 0.1 & - & 0.1 \\
\hline$\alpha$-Terpinene & $\mathrm{mh}$ & 1017 & 0.5 & - & 1.3 & 1.2 & 1.1 & 1.0 & 1.2 & 1.1 & 1.0 & 1.0 \\
\hline$p$-Cymene & $\mathrm{mh}$ & 1025 & 2.0 & 1.1 & 13.6 & 6.3 & 8.3 & 9.2 & 10.6 & 10.8 & 10.0 & 9.7 \\
\hline$\beta$-Phellandrene & $\mathrm{mh}$ & 1030 & 1.3 & - & - & - & - & - & - & - & - & - \\
\hline 1,8-Cineol & om & 1031 & - & 3.3 & - & - & 1.2 & 2.5 & - & 1.7 & 1.6 & 1.3 \\
\hline Limonene & $\mathrm{mh}$ & 1033 & 2.0 & - & 2.0 & 1.6 & 0.8 & - & 1.8 & - & - & - \\
\hline$(Z)-\beta$-Ocimene & $\mathrm{mh}$ & 1037 & - & - & 0.4 & - & - & - & 0.3 & 0.3 & 0.2 & 0.2 \\
\hline$(E)-\beta$-Ocimene & $\mathrm{mh}$ & 1050 & - & - & 0.1 & - & - & - & - & - & - & - \\
\hline$\gamma$-Terpinene & $\mathrm{mh}$ & 1060 & - & - & 6.4 & 1.8 & 4.7 & - & - & - & - & - \\
\hline p-Mentha-2.4(8)-diene & $\mathrm{mh}$ & 1088 & - & - & & 0.3 & & - & - & - & - & - \\
\hline Terpinolene & $\mathrm{mh}$ & 1089 & - & - & 0.3 & - & 0.2 & 0.3 & 0.3 & 0.3 & 0.3 & 0.3 \\
\hline Linalool & om & 1097 & 5.8 & 0.8 & 1.7 & 9.1 & 1.7 & 1.7 & 4.1 & 2.9 & 1.3 & 3.0 \\
\hline$\alpha$-Pinene oxide & om & 1099 & - & 1.3 & - & - & - & 0.4 & - & - & 0.2 & - \\
\hline$\alpha$-Campholenal & om & 1126 & - & 0.3 & - & - & - & - & - & - & - & - \\
\hline trans-Pinocarveol & om & 1139 & - & 0.5 & - & - & - & 0.1 & - & - & - & - \\
\hline cis-Verbenol & om & 1141 & - & 1.5 & - & - & - & - & - & - & - & - \\
\hline trans-Verbenol & om & 1145 & - & - & - & - & - & 0.3 & - & - & 0.2 & - \\
\hline Camphor & om & 1146 & - & 0.2 & 0.3 & - & - & 0.1 & 0.1 & 0.1 & - & - \\
\hline Hexyl isobutyrate & $\mathrm{nt}$ & 1152 & - & 1.2 & - & - & - & - & - & - & - & - \\
\hline 2-Methylbutyl angelate & $\mathrm{nt}$ & 1158 & - & 0.4 & - & - & - & 0.2 & - & - & - & - \\
\hline Borneol & om & 1169 & - & - & 2.7 & 0.6 & 0.4 & 1.3 & 1.4 & 1.5 & 1.1 & 0.9 \\
\hline 4-Terpineol & om & 1177 & 0.5 & - & 1.5 & 1.0 & 1.2 & 1.0 & 1.1 & 1.0 & 0.9 & 0.9 \\
\hline$\alpha$-Terpineol & om & 1189 & 1.2 & 0.4 & 0.9 & 0.6 & 0.3 & 0.6 & 0.7 & 0.9 & 0.3 & 0.4 \\
\hline Dihydrocarvacrol & om & 1194 & - & - & - & - & - & 0.2 & 0.2 & - & 0.2 & 0.2 \\
\hline Myrtinol & om & 1196 & - & 0.3 & - & - & - & - & - & - & - & - \\
\hline Verbenone & om & 1205 & - & 0.4 & - & - & - & - & - & - & - & - \\
\hline trans-Carveol & om & 1217 & - & 0.5 & - & - & - & - & - & - & - & - \\
\hline$\gamma$-Terpineol & om & 1218 & - & - & 0.4 & - & - & - & - & - & - & - \\
\hline (Z)-Cinnamaldehyde & $\mathrm{nt}$ & 1219 & 3.1 & - & - & - & - & 0.2 & - & 0.2 & - & 0.1 \\
\hline Nerol & om & 1230 & - & 0.2 & - & - & - & - & - & - & - & - \\
\hline cis-Sabinene hydrate & om & 1240 & - & - & 0.3 & 0.6 & 0.2 & 0.2 & 0.3 & 0.2 & 0.2 & 0.2 \\
\hline Cuminaldehyde & om & 1242 & - & - & 5.1 & - & - & 2.2 & 2.0 & 2.3 & 1.8 & 1.2 \\
\hline Carvone & om & 1243 & - & 0.2 & 0.2 & - & 0.2 & - & 0.1 & 0.1 & - & 0.2 \\
\hline (Z)-3-Hexenyl isovalerate & nt & 1245 & - & 0.3 & - & - & - & - & - & - & - & - \\
\hline trans-myrtanol & om & 1261 & - & - & - & - & - & - & - & - & - & - \\
\hline Geranial & om & 1267 & - & - & - & - & 0.2 & - & - & - & - & - \\
\hline (E)-cinnamaldehyde & nt & 1270 & 55.3 & - & - & - & - & 5.0 & 6.1 & 10.4 & - & 5.8 \\
\hline hexyl angelate & nt & 1286 & - & 0.8 & - & - & - & 0.6 & - & - & - & - \\
\hline isobornyl acetate & om & 1286 & - & - & 0.2 & - & - & - & - & - & - & - \\
\hline Thymol & om & 1290 & - & - & 7.0 & 2.4 & 0.5 & 3.0 & 3.3 & 3.0 & 2.8 & 2.1 \\
\hline
\end{tabular}


Table 3 (continued)

\begin{tabular}{|c|c|c|c|c|c|c|c|c|c|c|c|c|}
\hline \multirow[t]{2}{*}{ Compound } & \multirow[t]{2}{*}{ Class } & \multirow[t]{2}{*}{ LRI } & \multicolumn{5}{|l|}{ Eos } & \multicolumn{5}{|c|}{ Mixture of EOs } \\
\hline & & & $C z$ & $H i$ & $S m$ & $T s$ & $T c$ & A & $\mathrm{B}$ & $\mathrm{C}$ & $\mathrm{D}$ & $\mathrm{E}$ \\
\hline Carvacrol & om & 1299 & - & - & 44.5 & 69.3 & 70.5 & 39.9 & 52.3 & 46.7 & 54.7 & 59.2 \\
\hline neryl formate & om & 1307 & - & 0.2 & - & - & - & - & - & - & - & - \\
\hline Eugenol & $\mathrm{pp}$ & 1359 & - & - & - & - & - & 0.4 & 0.4 & 0.9 & 0.9 & 0.5 \\
\hline neryl acetate & om & 1362 & - & 6.2 & - & - & - & - & - & - & - & - \\
\hline cyclosativene & $\mathrm{sh}$ & 1371 & - & - & - & - & - & 0.1 & - & - & - & - \\
\hline geranyl acetate & om & 1372 & - & - & 0.1 & - & - & - & - & - & - & - \\
\hline linalool isobutyrate & om & 1374 & - & - & - & - & - & 1.2 & - & - & - & - \\
\hline$\alpha$-copaene & sh & 1377 & 1.7 & - & - & - & - & 1.0 & 0.4 & 0.6 & 0.8 & 0.3 \\
\hline Daucene & sh & 1382 & - & 4.1 & - & - & - & - & - & - & - & - \\
\hline iso-italicene & sh & 1402 & - & 6.0 & - & - & - & 1.1 & - & - & 0.9 & - \\
\hline cis- $\alpha$-bergamotene & sh & 1413 & - & 1.5 & - & - & - & 0.3 & - & - & 0.2 & - \\
\hline$\beta$-caryophyllene & sh & 1419 & 8.7 & 0.2 & 2.6 & 1.1 & 2.7 & 2.6 & 2.6 & 3.4 & 2.2 & 2.7 \\
\hline trans- $\alpha$-bergamotene & sh & 1435 & - & 1.4 & - & - & - & 0.3 & - & - & 0.2 & - \\
\hline$\alpha$-guaiene & sh & 1444 & - & - & 0.2 & 0.3 & - & - & - & - & - & - \\
\hline 8-decene-3,5-dione,4,6,9-trimethyl- & $\mathrm{nt}$ & 1449 & - & - & - & - & - & - & - & - & - & - \\
\hline neryl propanoate & om & 1455 & - & 1.0 & - & - & - & 0.2 & - & - & 0.3 & - \\
\hline$\alpha$-humulene & $\mathrm{sh}$ & 1455 & 3.8 & - & 0.1 & - & - & 0.4 & 0.4 & 0.6 & - & 0.5 \\
\hline allo-aromadendrene & sh & 1460 & - & - & - & - & - & 0.1 & - & - & - & - \\
\hline dehydro-aromadendrene & sh & 1465 & - & - & - & - & - & - & - & 0.1 & - & - \\
\hline$\alpha$-acoradiene & sh & 1466 & - & 0.6 & - & - & - & 0.1 & - & - & - & - \\
\hline$\beta$-acoradiene & $\mathrm{sh}$ & 1466 & - & 0.9 & 0.1 & - & - & 0.1 & - & - & - & - \\
\hline$\gamma$-muurolene & sh & 1480 & - & 2.6 & - & - & - & - & - & - & - & - \\
\hline ar-curcumene & $\mathrm{sh}$ & 1481 & 0.1 & - & - & - & - & 3.4 & - & - & - & - \\
\hline$\gamma$-himachelene & sh & 1483 & - & - & - & - & - & 0.6 & - & - & 0.1 & - \\
\hline$\beta$-selinene & $\mathrm{sh}$ & 1490 & - & - & 0.2 & 0.2 & - & 1.1 & 0.1 & 0.1 & 3.4 & 0.1 \\
\hline viridiflorene & sh & 1497 & - & 18.9 & - & - & - & 0.9 & - & - & 0.7 & - \\
\hline$\alpha$-muurolene & sh & 1500 & - & - & - & - & - & 0.2 & - & - & 0.1 & - \\
\hline$\beta$-bisabolene & sh & 1506 & - & 0.4 & 0.7 & - & 0.4 & 0.5 & 0.3 & 0.4 & 0.5 & 0.3 \\
\hline$\alpha$-bulnesene & sh & 1510 & - & 1.9 & - & - & - & - & - & - & - & - \\
\hline Cubebol & os & 1515 & - & 1.7 & - & - & - & - & - & - & - & - \\
\hline$\beta$-curcumene & sh & 1516 & - & - & 0.2 & - & - & - & - & - & - & - \\
\hline$\alpha$-cadinene & $\mathrm{sh}$ & 1539 & - & 0.2 & - & - & - & - & - & - & - & - \\
\hline caryophyllene oxide & os & 1583 & 0.9 & - & 0.7 & 0.1 & 1.3 & 1.8 & 0.5 & 0.7 & 1.6 & 0.7 \\
\hline Globulol & os & 1585 & - & 1.1 & - & - & - & - & - & - & - & - \\
\hline humulene oxide II & os & 1608 & - & 0.5 & - & - & - & - & - & - & - & - \\
\hline tetradecanal & $\mathrm{nt}$ & 1613 & 0.3 & - & - & - & - & - & - & - & - & - \\
\hline cis-cadin-4-en-7-ol & os & 1637 & - & 0.3 & - & - & - & - & - & - & - & - \\
\hline 14-hydroxy-9-epi-(E)-caryophyllene & os & 1670 & - & - & - & - & - & 0.2 & - & - & - & - \\
\hline$(Z, E)$-farnesol & os & 1690 & - & 0.3 & - & - & - & - & - & - & - & - \\
\hline benzyl benzoate & $\mathrm{nt}$ & 1760 & 2.3 & - & - & - & - & - & 0.1 & 0.4 & - & 0.3 \\
\hline dirm-8-en-7-one & os & 1763 & - & 1.1 & - & - & - & - & - & - & - & - \\
\hline$\alpha$-bisabolol acetate & os & 1798 & - & 0.4 & - & - & - & - & - & - & - & - \\
\hline sesquilavandulyl acetate & os & 1809 & - & 0.6 & - & - & - & - & - & - & - & - \\
\hline \multicolumn{13}{|l|}{ Class of compounds } \\
\hline Monoterpene Hydrocarbons (mh) & & & 8.9 & 26.8 & 28.7 & 14.1 & 17.9 & 18.2 & 18.1 & 16.3 & 17.0 & 14.4 \\
\hline Nom-terpene derivatives (nt) & & & 61.2 & 5.4 & 0.6 & 0.2 & 0.2 & 6.2 & 6.6 & 11.4 & 0.3 & 6.5 \\
\hline Oxygenated Monoterpenes (om) & & & 7.5 & 17.6 & 65.0 & 83.6 & 76.4 & 54.9 & 65.6 & 60.4 & 65.6 & 69.6 \\
\hline Oxygenated Sesquiterpenes (os) & & & 0.9 & 5.7 & 0.7 & 0.1 & 1.3 & 2.0 & 0.5 & 0.7 & 1.6 & 0.7 \\
\hline
\end{tabular}


Table 3 (continued)

\begin{tabular}{|c|c|c|c|c|c|c|c|c|c|c|c|c|}
\hline \multirow[t]{2}{*}{ Compound } & \multirow[t]{2}{*}{ Class } & \multirow[t]{2}{*}{ LRI } & \multicolumn{5}{|l|}{ Eos } & \multicolumn{5}{|c|}{ Mixture of EOs } \\
\hline & & & $C z$ & $H i$ & $S m$ & $T s$ & $T c$ & A & $\mathrm{B}$ & $\mathrm{C}$ & $\mathrm{D}$ & $\mathrm{E}$ \\
\hline Phenylpropanoids (pp) & & & 4.1 & - & - & - & - & 0.4 & 0.4 & 0.9 & 0.9 & 0.5 \\
\hline Sesquiterpene Hydrocarbons (sh) & & & 14.3 & 39.0 & 4.1 & 1.6 & 3.1 & 12.8 & 3.8 & 5.2 & 9.1 & 4.1 \\
\hline Total Identified & & & 96.9 & 94.8 & 99.1 & 99.6 & 98.9 & 94.5 & 95.0 & 94.9 & 94.5 & 95.8 \\
\hline
\end{tabular}

LRI linear retention indices

Single EO: Cinnamomum zeylanicum (Cz), Helichrysum italicum (Hi), Satureja montana (Sm), Thymus capitatus (Tc) and Thymus serpyllum $(T s)$

Blends: A-Sm 30\%, Tc 30\%, Hi 25\%, Cz 15\%; B-Sm 30\%, Ts 25\%, Tc 25\%, Cz 20\%; C-Sm 34\%, Cz 34\%, Tc 22\%, Ts 10\%; D-Tc 50\%, Sm $30 \%$, Hi 20\%; E-Tc 40\%, Cz 20\%, Sm 20\%, Ts 20\%

Table 4 MIC and MBC mode values of single essential oil and blends

\begin{tabular}{|c|c|c|c|c|c|c|c|c|c|c|}
\hline \multirow{2}{*}{$\begin{array}{l}\text { Single EO- } \\
\text { EOs blend }\end{array}$} & \multicolumn{5}{|c|}{ MIC mode $(\mathrm{g} / \mathrm{ml})$} & \multicolumn{5}{|c|}{ MBC mode $(\mathrm{g} / \mathrm{ml})$} \\
\hline & Sa1 & $\mathrm{Sa} 2$ & $S x 1$ & $S \times 2$ & $S \times 3$ & $S a 1$ & $\mathrm{Sa} 2$ & $S x 1$ & $S \times 2$ & $S \times 3$ \\
\hline$C z$ & 0.94 & 0.94 & 1.88 & 1.88 & 1.88 & 3.75 & 3.75 & 3.75 & 3.75 & 3.75 \\
\hline$H i$ & 7.37 & 7.37 & 7.37 & 14.73 & 14.73 & 29.47 & 29.47 & 29.47 & 58.94 & 58.94 \\
\hline$S m$ & 3.69 & 3.69 & 7.38 & 7.38 & 3.69 & 7.38 & 7.38 & 7.38 & 14.76 & 7.38 \\
\hline$T s$ & 1.9 & 3.8 & 3.8 & 3.8 & 3.8 & 1.9 & 7.61 & 7.61 & 7.61 & 7.61 \\
\hline$T c$ & 1.86 & 1.86 & 1.86 & 3.71 & 3.71 & 1.86 & 1.86 & 1.86 & 7.42 & 7.42 \\
\hline A & 0.46 & 0.93 & 0.93 & 0.93 & 0.46 & 0.93 & 0.93 & 0.93 & 1.85 & 0.46 \\
\hline B & 1.84 & 1.84 & 1.84 & 1.84 & 0.92 & 1.84 & 1.84 & 1.84 & 1.84 & 1.84 \\
\hline $\mathrm{C}$ & 0.47 & 0.47 & 0.47 & 0.47 & 0.24 & 0.95 & 0.47 & 0.47 & 0.47 & 0.47 \\
\hline D & 0.94 & 0.94 & 0.94 & 0.94 & 0.47 & 0.94 & 0.94 & 0.94 & 0.94 & 0.94 \\
\hline E & 0.47 & 0.47 & 0.47 & 0.47 & 0.47 & 0.47 & 0.47 & 0.47 & 0.47 & 0.47 \\
\hline
\end{tabular}

Staphylococcus aureus isolates: $S a 1$ and $S a 2$. Staphylococcus xylosus isolates: $S x 1, S x 2$ and $S x 3$

Single EO: Cinnamomum zeylanicum $(\mathrm{Cz})$, Helichrysum italicum (Hi), Satureja montana (Sm), Thymus capitatus (Tc) and Thymus serpyllum (Ts)

Blends: A-Sm 30\%, Tc 30\%, Hi 25\%, Cz 15\%; B-Sm 30\%, Ts 25\%, Tc 25\%, Cz 20\%; C-Sm 34\%, Cz $34 \%$, Tc 22\%, Ts 10\%; D-Tc 50\%, Sm 30\%, Hi 20\%; E-Tc 40\%, Cz 20\%, Sm 20\%, Ts $20 \%$
The corresponding MBC mode values for each of these EOs coincided with MIC mode values or settled one dilution step forward. These results are in accordance with the relevant literature, which states that essential oils obtained from Lamiaceae plants often show a marked antibacterial activity due to the presence of thymol, carvacrol and its precursor p-cymene (Hyldgaard et al. 2012; Rajput et al. 2018).

Likewise, the broad-spectrum antibacterial activity of Cinnamon bark essential oil is widely documented and has been attributed to the presence of cinnamaldehyde (Ananda Baskaran et al. 2009; Dal Pozzo et al. 2012; Friedman 2017).

Fewer studies have been conducted on the antibacterial activity of Manuka essential oil, which has remarkable antimicrobial properties referable to the presence of some bioactive compounds, such as leptospermone and iso-leptospermone (Lis-Balchin et al. 2000; Fratini et al. 2017).

A second group of essential oils used in the screening test demonstrated an intermediate level of antibacterial activity: Helichrysum italicum and Origanum majorana (mode values between 1:64 v/v and 1:128 v/v); Cistus ladanifer, Litsea cubeba and Ocimum basilicum (mode values between 1:32 v/v and 1:128 v/v); Citrus medica, Cuminum cyminum, Lavandula officinalis, Rosa $\times$ damascene, Rosmarinus officinalis, Rosmarinus officinalis ct cineole (mode values between 1:32 v/v and 1:64 v/v); Citrus $\times$ bergamia, Citrus limon, Coriandrum sativum, Elettaria cardamomum, Juniperus communis (mode values of 1:32 v/v). It is important to note that the MBC mode values for all the above mentioned EOs did not follow the same trend as the first group, in some cases deviating by two dilutions in comparison to MIC values. However, the wide variability in antimicrobial activity showed by this second group is reported in several studies (Chao et al. 2008; Giovannini et al. 2016; Imane et al. 2020; Mollova et al. 2020). The remaining EOs selected for initial screening test showed mild antibacterial activity against isolated staphylococci.

GC-EIMS determination of the five chosen EOs and their blends are reported in Table 3. The three Lamiaceae EOs 
were characterised by the presence of $p$-cymene, $\boldsymbol{\gamma}$-terpinene and carvacrol. These compounds were found, respectively, in Satureja montana EO at 13.6\%, 6.4\%, 44.5\%, in Thymus serpyllum at $6.3 \%, 1.8 \%, 69.3 \%$ and in Thymus capitatus at $8.3 \%, 4.7 \%$ and $70.5 \%$. Thymol was present in a higher percentage in Satureja montana EO (7.0\%), together with cuminaldehyde (5.1\%). Linalool, identified in Cinnamomum zeylanicum (5.8\%) and Thymus serpyllum (9.1\%), is a monoterpene also present in EOs obtained from lavender, basil, laurel, bergamot, jasmine, mandarin leaves, orange leaves, lemon leaves and many other plants; this compound interferes with the integrity and functionality of the bacterial cell membrane, causing alterations in membrane potential and consequent loss of cytoplasmic material (Silva et al. 2015; Greay and Hammer 2015).

The predominant compound in cinnamon-bark EO was the $(E)$ isomer of cinnamaldehyde $(55.3 \%)$ followed by $\beta$-cariophyllene $(8.7 \%)$, a sesquiterpene which has demonstrated both a selective antibacterial activity against $S$. aureus and a marked antioxidant activity (Dorman and Deans 2000; Dahham et al. 2015). The main molecules identified in everlasting flowers EO were $\alpha$-pinene (24.6\%) and viridiflorene (18.9\%). The former is a monoterpene with proven antibacterial activity (de Sousa Eduardo et al. 2018; Ložienè et al. 2018) while the latter is a sesquiterpene produced by the dehydration of viridiflorol whose biological activities are not yet well known, firstly isolated from Majorana hortensis EO (Taskinen 1974) and then also found in Melaleuca alternifolia EO (Swords and Hunter 1978).

Interestingly, we want to highlight that the mixtures' GCEIMS showed different compounds percentages compared to the one expected by calculation based on the single EOs composition. These values differ significantly especially in the case of carvacrol (A: $+5.38 \%$; B: $+4.00 \%$; C: $+9.10 \%$; $\mathrm{D}:+6.08 \%$; $\mathrm{E}:+8.18 \%)$ and the trans isomer of cinnamaldehyde (A: $-3.32 \%$; B: $-4.96 \%$; C: $-8.35 \%$; E: $-5.24 \%$ ). This discrepancy in data could be ascribed to oxidative phenomena affecting the numerous carvacrol precursors present in some EOs and degradation of cinnammaldehyde to benzaldehyde, a non-volatile compound not detectable by gas chromatographic analysis (Wang et al. 2009). Major deviations could be, therefore, caused by chemical phenomena occurring between the various components of EOs. All these suggests emphasize that combination of different phytocomplexes could determine dynamic and mutable mixture, leading to reciprocal modifications through interactions of their several compounds.

MIC and MBC values of both single EOs and their blends are reported in Table 4. Whereas the behaviour of the two strains of $S$. aureus was similar with respect to the selected EOs and their mixtures. S. xylosus showed slight differences in terms of susceptibility. The five mixtures tested showed a strong synergistic activity among the essential oils. Comparing the MIC mode values obtained from individual oils and those obtained from the mixtures, it is evident that lower concentrations of each mixture were required to inhibit staphylococci isolates. Even if compared to $C z \mathrm{EO}$, which showed the highest antibacterial activity amongst the single EOs (MIC range from 0.94 $\mathrm{g} / \mathrm{ml}$ to $1.88 \mathrm{~g} / \mathrm{ml}$ ), each of the blends reported a lower MIC range (A: MIC range from $0.46 \mathrm{~g} / \mathrm{ml}$ to $0.93 \mathrm{~g} / \mathrm{ml}$; B: MIC range from $0.92 \mathrm{~g} / \mathrm{ml}$ to $1.84 \mathrm{~g} / \mathrm{ml}$; C: MIC range from $0.24 \mathrm{~g} / \mathrm{ml}$ to $0.47 \mathrm{~g} / \mathrm{ml}$; D: MIC range from $0.47 \mathrm{~g} / \mathrm{ml}$ to $0.94 \mathrm{~g} / \mathrm{ml}$; E: MIC range equal to $0.47 \mathrm{~g} / \mathrm{ml}$ ).

MBC values of blends followed the same trend observed for the single EOs. The best results were obtained from mixture E with MIC and MBC mode values of $0.47 \mathrm{~g} / \mathrm{ml}$ for each strain tested. Mixture B reported a MIC range between $0.92 \mathrm{~g} / \mathrm{ml}$ and $1.84 \mathrm{~g} / \mathrm{ml}$ proving to be the less effective of the mixtures, but still slightly more active than the best single EO used in the tests. $C z, S m, T c$ and $T s$ EOs showed a strong antibacterial activity against $S$. aureus strains, that was less marked for $\mathrm{Hi}$ EO. Mixtures B, C and D were more effective on S. xylosus strains, while mixtures $\mathrm{A}$ and $\mathrm{E}$ showed equal activity against both the staphylococci species.

\section{Conclusions}

Both individually tested EOs and their blends proved to have in vitro antibacterial properties, particularly remarkable for the latter. This is ascribable to the synergistic effect between different essential oils, allowing to reduce the EO percentage in the formulation of mixtures and limiting the risk of side effects associated with the use of these substances. Synergy should be more thoroughly investigated by determining FIC (fractional inhibitory concentration) and FBC (fractional bactericidal concentration) values at the same time as those of MIC and MBC concerning single EOs. On the other hand, this type of analysis turns out to be very laborious and expensive for the high amount of essential oil needed, especially using mixtures with more than three different EOs. On the basis of these in vitro positive results, it would therefore be advisable to assess the effectiveness and safety of these substances in vivo, so that essential oils and their appropriate mixtures may hereafter represent a real therapeutic option, as an alternative or complement to traditional antibiotic therapy.

Acknowledgements The authors want to thank Mario Rosario Rizzi (FLORA s.r.l.®) for the essential oil samples provided and Stefano Spinelli for the effective cooperation, both essential for carrying out the research. 
Funding Open access funding provided by Università di Pisa within the CRUI-CARE Agreement.

Open Access This article is licensed under a Creative Commons Attribution 4.0 International License, which permits use, sharing, adaptation, distribution and reproduction in any medium or format, as long as you give appropriate credit to the original author(s) and the source, provide a link to the Creative Commons licence, and indicate if changes were made. The images or other third party material in this article are included in the article's Creative Commons licence, unless indicated otherwise in a credit line to the material. If material is not included in the article's Creative Commons licence and your intended use is not permitted by statutory regulation or exceeds the permitted use, you will need to obtain permission directly from the copyright holder. To view a copy of this licence, visit http://creativecommons.org/licenses/by/4.0/.

\section{References}

Ananda Baskaran S, Kazmer GW, Hinckley L et al (2009) Antibacterial effect of plant-derived antimicrobials on major bacterial mastitis pathogens in vitro. J Dairy Sci 92:1423-1429. https:// doi.org/10.3168/jds.2008-1384

Antonios Z, Theofilos P, Ioannis M et al (2015) Prevalence, genetic diversity, and antimicrobial susceptibility profiles of Staphylococcus aureus isolated from bulk tank milk from Greek traditional ovine farms. Small Rumin Res 125:120-126. https://doi. org/10.1016/j.smallrumres.2015.02.009

Antunes Viegas D, Palmeira-de-Oliveira A, Salgueiro L et al (2014) Helichrysum italicum: From traditional use to scientific data. J Ethnopharmacol 151:54-65. https://doi.org/10.1016/j.jep.2013. 11.005

Barceloux DG (2009) Cinnamon (Cinnamomum Species). Dis Mon $55: 327-335$

Bergonier D, de Crémoux R, Rupp R et al (2003) Mastitis of dairy small ruminants. Vet Res 34:689-716. https://doi.org/10.1051/ vetres:2003030

Cannas E, Dore S, Lollai S, Liciardi M (2019) Le mastiti dei piccoli ruminanti, 3rd edn. Istituto Zooprofilattico Sperimentale della Sardegna "G. Pegreffi"

Caprioli G, Lupidi G, Maggi F (2019) Comparison of chemical composition and antioxidant activities of two Winter savory subspecies (Satureja montana subsp. variegata and Satureja montana subsp. montana) cultivated in Northern Italy. Nat Prod Res 33:3143-3147. https://doi.org/10.1080/14786419.2018.1516661

Chao S, Young G, Oberg C, Nakaoka K (2008) Inhibition of methicillin-resistant Staphylococcus aureus (MRSA) by essential oils. Flavour Fragr J 23:444-449. https://doi.org/10.1002/ffj. 1904

Clinical and Laboratory Standards Institute (CLSI) (2013) Performance standards for antimicrobial susceptibility testing. CLSI, Wayne, PA

Cockerill III FR, Wikler MA, Alder J et al (2012) Performance standards for antimicrobial disk susceptibility tests; Approved standard, vol 32, no 1. CLSI (Clinical and Laboratory Standards Institute), Wayne, PA, p 58

Contreras A, Sierra D, Sánchez A et al (2007) Mastitis in small ruminants. Small Rumin Res 68:145-153. https://doi.org/10. 1016/j.smallrumres.2006.09.011

Dahham S, Tabana Y, Iqbal M et al (2015) The anticancer, antioxidant and antimicrobial properties of the sesquiterpene $\beta$-Caryophyllene from the essential oil of Aquilaria crassna. Molecules 20:11808 11829. https://doi.org/10.3390/molecules200711808
Dal Pozzo M, Loreto ÉS, Santurio DF et al (2012) Antibacterial activity of essential oil of cinnamon and trans-cinnamaldehyde against Staphylococcus spp. Isolated from clinical mastitis of cattle and goats. Acta Sci Vet 40:1-5

Demir A, Mercanoglu Taban B, Aslan M et al (2009) Antimicrobial effect of Helichrysum plicatum subsp. plicatum. Pharm Biol 47:289-297. https://doi.org/10.1080/13880200802590434

Djihane B, Mihoub ZM (2016) Evaluation of in vitro anti-inflammatory activity of Helichrysum italicum (Roth) G. Don essential oil Der Pharm Lett 8:41-44

Dorman HJD, Deans SG (2000) Antimicrobial agents from plants: antibacterial activity of plant volatile oils. J Appl Microbiol 88:308-316. https://doi.org/10.1046/j.1365-2672.2000.00969.x

European Committee on Antimicrobial Susceptibility Testing (EUCAST) (2017) Breakpoint tables for interpretation of MICs and zone diameters, Version 7.1

Fratini F, Casella S, Leonardi M et al (2014) Antibacterial activity of essential oils, their blends and mixtures of their main constituents against some strains supporting livestock mastitis. Fitoterapia 96:1-7. https://doi.org/10.1016/j.fitote.2014.04.003

Fratini F, Mancini S, Turchi B et al (2017) A novel interpretation of the Fractional Inhibitory Concentration Index: The case Origanum vulgare L. and Leptospermum scoparium J. R. et G. Forst essential oils against Staphylococcus aureus strains. Microbiol Res 195:11-17. https://doi.org/10.1016/j.micres.2016.11.005

Fratini F, Mancini S, Turchi B et al (2019) Antimicrobial activity of three essential oils (cinnamon, manuka, and winter savory), and their synergic interaction, against Listeria monocytogenes. Flavour Fragr J 34:339-348. https://doi.org/10.1002/ffj.3514

Friedman M (2017) Chemistry, antimicrobial mechanisms, and antibiotic activities of cinnamaldehyde against pathogenic bacteria in animal feeds and human foods. J Agric Food Chem 65:1040610423. https://doi.org/10.1021/acs.jafc.7b04344

Gaio V, Lima CA, Oliveira F et al (2017) Carvacrol is highly disruptive against coagulase-negative staphylococci in in vitro biofilms. Future Microbiol 12:1487-1496. https://doi.org/10.2217/ fmb-2017-0122

Giovannini D, Gismondi A, Basso A et al (2016) Lavandula angustifolia mill. Essential oil exerts antibacterial and anti-inflammatory effect in macrophage mediated immune response to Staphylococcus aureus. Immunol Invest 45:11-28. https://doi.org/10.3109/ 08820139.2015.1085392

Greay SJ, Hammer KA (2015) Recent developments in the bioactivity of mono- and diterpenes: anticancer and antimicrobial activity. Phytochem Rev 14:1-6. https://doi.org/10.1007/ s11101-011-9212-6

Hyldgaard M, Mygind T, Meyer RL (2012) Essential oils in food preservation: mode of action, synergies, and interactions with food matrix components. Front Microbiol 3:1-24. https://doi.org/10. 3389/fmicb.2012.00012

Imane NI, Fouzia H, Azzahra LF et al (2020) Chemical composition, antibacterial and antioxidant activities of some essential oils against multidrug resistant bacteria. Eur J Integr Med 35:101074. https://doi.org/10.1016/j.eujim.2020.101074

Jafari F, Ghavidel F, Zarshenas MM (2016) A critical overview on the pharmacological and clinical aspects of popular Satureja Species. J Acupunct Meridian Stud 9:118-127. https://doi.org/10.1016/j. jams.2016.04.003

Jarić S, Mitrović M, Pavlović P (2015) Review of ethnobotanical, phytochemical, and pharmacological study of Thymus serpyllum L. Evidence-Based Complement Altern Med 2015:1-10. https://doi. org/10.1155/2015/101978

Lis-Balchin M, Hart SL, Deans SG (2000) Pharmacological and antimicrobial studies on different tea-tree oils (Melaleuca alternifolia, Leptospermum scoparium or Manuka and Kunzea ericoides or Kanuka), originating in Australia and New Zealand. Phyther Res 
14:623-629. https://doi.org/10.1002/1099-1573(200012)14:8\% 3c623::AID-PTR763\%3e3.0.CO;2-Z

Lisows-Łkaysiak K, Dudko P, Kosecka-Strojek M et al (2018) Characteristics of advanced methods used for typing bacterial isolates from mastitis with particular reference to Staphylococci. Pol J Vet Sci 21:229-239. https://doi.org/10.24425/119041

Lorenzi V, Muselli A, Bernardini AF et al (2009) Geraniol restores antibiotic activities against multidrug-resistant isolates from gram-negative species. Antimicrob Agents Chemother 53:22092211. https://doi.org/10.1128/AAC.00919-08

Ložienė K, Švedienė J, Paškevičius A et al (2018) Influence of plant origin natural $\alpha$-pinene with different enantiomeric composition on bacteria, yeasts and fungi. Fitoterapia 127:20-24. https://doi. org/10.1016/j.fitote.2018.04.013

Mahadlek J, Charoenteeraboon J, Phaechamud T (2012) Combination effects of the antimicrobial agents and cinnamon oil. Adv Mater Res 506:246-249. https://doi.org/10.4028/www.scientific.net/ AMR.506.246

Maissa BJ, Walid H (2015) Antifungal activity of chemically different essential oils from wild Tunisian Thymus spp. Natural Product Res 29:869-873

Maksimovic S, Tadic V, Skala D, Zizovic I (2017) Separation of phytochemicals from Helichrysum italicum : An analysis of different isolation techniques and biological activity of prepared extracts. Phytochemistry 138:9-28. https://doi.org/10.1016/j.phytochem. 2017.01.001

Marassi CG, Rossi A (2015) Phytotherapeutic Approaches. In: Katsambas AD, Lotti TM, Dessinioti C, D’Erme AM (eds) European Handbook of Dermatological Treatments. Springer, Berlin , pp 1481-1485

Marin M, Novakovic M, Vuckovic I et al (2018) Wild Thymus capitatus Hoff. Et Link. chemical composition, antioxidant and antimicrobial activities of the essential oil. J Essent Oil Bear Plants 21:388-399. https://doi.org/10.1080/0972060X.2018.1449668

Mollova S, Fidan H, Antonova D et al (2020) Chemical composition and antimicrobial and antioxidant activity of Helichrysum italicum (Roth) G.Don subspecies essential oils. Turkish J Agric For 44:371-378. https://doi.org/10.3906/tar-1909-34

Mork T, Tollersrud T, Kvitle B et al (2005) Comparison of Staphylococcus aureus genotypes recovered from cases of bovine, ovine, and caprine mastitis. J Clin Microbiol 43:3979-3984. https://doi. org/10.1128/JCM.43.8.3979-3984.2005

Nabavi SM, Marchese A, Izadi M et al (2015) Plants belonging to the genus Thymus as antibacterial agents: From farm to pharmacy. Food Chem 173:339-347. https://doi.org/10.1016/j.foodchem. 2014.10.042

Paaver U, Orav A, Arak E et al (2008) Phytochemical analysis of the essential oil of Thymus serpyllum L. growing wild in Estonia. Nat Prod Res 22:108-115. https://doi.org/10.1080/1478641060 1035118

Pisseri F, Bertoli A, Pistelli L (2008) Essential oils in medicine: principles of therapy. Parassitologia 50:89-91

Pistelli L, Najar B, Giovanelli S et al (2017) Agronomic and phytochemical evaluation of lavandin and lavender cultivars cultivated in the Tyrrhenian area of Tuscany (Italy). Ind Crops Prod 109:3744. https://doi.org/10.1016/j.indcrop.2017.07.041

Pyorala S, Taponen S (2009) Coagulase-negative staphylococciEmerging mastitis pathogens. Vet Microbiol 134:3-8. https://doi. org/10.1016/j.vetmic.2008.09.015

Rajput JD, Bagul SD, Pete UD et al (2018) Perspectives on medicinal properties of natural phenolic monoterpenoids and their hybrids. Mol Divers 22:225-245. https://doi.org/10.1007/ s11030-017-9787-y

Saleem M, Bhatti HN, Jilani MI, Hanif MA (2015) Bioanalytical evaluation of Cinnamomum zeylanicum essential oil. Nat Prod Res 29:1857-1859. https://doi.org/10.1080/14786419.2014.1002088 dos Santos Rodrigues JB, de Carvalho RJ, de Souza NT et al (2017) Effects of oregano essential oil and carvacrol on biofilms of Staphylococcus aureus from food-contact surfaces. Food Control 73:1237-1246. https://doi.org/10.1016/j.foodcont.2016.10. 043

Sharma C, Rokana N, Chandra M et al (2018) Antimicrobial resistance: its surveillance, impact, and alternative management strategies in dairy animals. Front Vet Sci 4:1-27. https://doi.org/10.3389/ fvets.2017.00237

Silva VA, Sousa JP, Guerra FQS et al (2015) Antibacterial activity of Ocimum basilicum essential oil and linalool on bacterial isolates of clinical importance. Int J Pharmacogn Phytochem Res 7:1066-1071

de Sousa EL, Farias TC, Ferreira SB et al (2018) Antibacterial activity and time-kill kinetics of positive enantiomer of $\alpha$-pinene against strains of Staphylococcus aureus and Escherichia coli. Curr Top Med Chem 18:917-924. https://doi.org/10.2174/ 1568026618666180712093914

Swords G, Hunter GLK (1978) Composition of Australian Tea Tree Oil (Melaleuca alternifolia). J Agric Food Chem 26:734-737

Taponen S, Nykäsenoja S, Pohjanvirta T et al (2015) Species distribution and in vitro antimicrobial susceptibility of coagulase-negative staphylococci isolated from bovine mastitic milk. Acta Vet Scand 58:12. https://doi.org/10.1186/s13028-016-0193-8

Tariq S, Wani S, Rasool W et al (2019) A comprehensive review of the antibacterial, antifungal and antiviral potential of essential oils and their chemical constituents against drug-resistant microbial pathogens. Microb Pathog 134:103580. https://doi.org/10.1016/j. micpath.2019.103580

Taskinen J (1974) Composition of the essential oil of sweet marjoram obtained by distillation with steam and by extraction and distillation with alcohol-water mixture. Acta Chem Scand B 28:1121-1128

Tepe B, Cilkiz M (2016) A pharmacological and phytochemical overview on Satureja. Pharm Biol 54:375-412. https://doi.org/10. 3109/13880209.2015.1043560

van Vuuren S, Orchard A, Viljoen A (2019) Essential oils: Fragrant pools of antimicrobial synergism explored. Synergy 9:100051. https://doi.org/10.1016/j.synres.2019.100051

Vanderhaeghen W, Piepers S, Leroy F et al (2015) Identification, typing, ecology and epidemiology of coagulase negative staphylococci associated with ruminants. Vet J 203:44-51. https://doi.org/ 10.1016/j.tvj1.2014.11.001

Wang R, Wang R, Yang B (2009) Extraction of essential oils from five cinnamon leaves and identification of their volatile compound compositions. Innov Food Sci Emerg Technol 10:289-292. https:// doi.org/10.1016/j.ifset.2008.12.002

Wendlandt S, Feßler AT, Monecke S et al (2013) The diversity of antimicrobial resistance genes among staphylococci of animal origin. Int J Med Microbiol 303:338-349. https://doi.org/10.1016/j.ijmm. 2013.02.006

Windria S, Widianingr DC, Salasia SIO (2016) Identification of Staphylococcus aureus and Coagulase Negative Staphylococci Isolates from Mastitis Milk of Etawa Crossbred Goat. Res J Microbiol 11:11-19. https://doi.org/10.3923/jm.2016.11.19

Publisher's Note Springer Nature remains neutral with regard to jurisdictional claims in published maps and institutional affiliations. 